\title{
CD40L in autoimmunity and mucosally induced tolerance
}

\section{Commentary}

See related article, pages $261-267$.

\author{
Mi-Na Kweon ${ }^{1}$ and Hiroshi Kiyono ${ }^{1,2}$ \\ ${ }^{1}$ Department of Mucosal Immunology, Research Institute for Microbial Diseases, Osaka University, Osaka, Japan \\ ${ }^{2}$ Immunobiology Vaccine Center, University of Alabama at Birmingham, Birmingham, Alabama, USA \\ Address correspondence to: Hiroshi Kiyono, Department of Mucosal Immunology, \\ Research Institute for Microbial Diseases, Osaka University, Osaka 565, Japan. \\ Phone: 81-6-6879-8292; Fax: 81-6-6878-6765; E-mail: kiyono@biken.osaka-u.ac.jp.
}

J. Clin. Invest. 109:171-173 (2002). DOI:10.1172/JCI200214930.

The administration of soluble antigens to the oral or nasal mucosa can lead to systemic unresponsiveness to a subsequent challenge with the same antigens (1). Such mucosally induced tolerance probably protects the body from hypersensitivity reactions to food proteins, pollen, and commensal bacterial antigens present in the normal mucosa and thus helps maintain an appropriate immunological balance between the host and its normal flora. Numerous molecular and cellular inhibitory mechanisms are involved in this unique and important immunological phenomenon. Several lines of evidence show that mucosally induced tolerance does not occur passively, by means of anergy, clonal deletion, or the simple absence of autoreactive immune cells, but also involves ignorance, receptor downregulation, or active cellular suppression (ref. 2; Figure 1a).

The fact that mucosally induced tolerance can suppress antigen-specific $\mathrm{T}$ cell proliferation, delayed-type hypersensitivity responses, and antibody production suggests attractive approaches for the prevention and control of autoimmune diseases, including multiple sclerosis, rheumatoid arthritis, encephalomyelitis, and type 1 diabetes (3). However, although mucosal administration of different autoantigens has been tested in human trials, this strategy has not yet yielded a successful therapy $(4,5)$. Some previous studies have suggested that this failure of human trials was due to concomitant induction of harmful cytotoxic $\mathrm{T}$ lymphocytes (CTLs) by mucosally administered antigen $(6,7)$.

In an attempt to overcome this obstacle, as reported in this issue of the JCI, Hänninen et al. (8) have induced a temporary blockade of the interaction between the costimulatory protein CD40 and its ligand CD40L. This interaction has been suggested to foster mucosally induced tolerance by several mechanisms, as shown in Figure 1a. In their present report, the authors show that blocking antibodies to $\mathrm{CD} 40 \mathrm{~L}$ can prevent the development of antigen-specific CTL responses without affecting the development of oral tolerance.

Hänninen and colleagues (8) tested this approach in rat insulin promotorOVA (RIP-OVA) mice, a diabetic transgenic strain in which the expression of the model antigen chicken ovalbumin (OVA) in the pancreatic $\beta$-islet cells provokes an autoimmune response to these cells. The consequent destruction of these islet cells thus provides a model for the events seen in type I diabetes. Oral administration of a high dose of OVA can promote mucosally induced tolerance, but this treatment also elicits CTL specific for OVA. However, Hänninen et al. observed that transient blockade of CD40L in this system prevented CTL responses without affecting mucosally induced tolerance toward OVA, either in wild-type or in RIP-OVA transgenic mice (Figure 1b). Furthermore, the severity of diabetes, as measured, for example, by blood glucose, was significantly improved after transient blockade of CD40L, suggesting that modulation of CD40L-CD40 interaction could be used clinically to uncouple the desired systemic, autoantigen-specific unresponsiveness from the oral induction of CTLs.

\section{Costimulation in mucosally induced tolerance}

These striking data thus appear to indicate that the CD40L-CD40 interaction is dispensable for mucosally induced tolerance. We suggest, however, that it would be premature to rule out a contribution of CD40L and other costimulatory factors to mucosally induced tolerance. In an earlier study, we found that systemic unresponsiveness to either of two model antigens (OVA or hen lysozyme) occurred in $\mathrm{CD}_{40 \mathrm{~L}^{+/+}}$wild-type controls but could not be induced in $C D 40 L^{-/-}$mice (9). Significantly, although these $C D 40 \mathrm{~L}^{-/-}$mice have multiple functional defects, such as a failure of germinal center formation, Ig class switching, and the inability to elicit Th1-type responses $(10,11)$, their antigen-specific $T$ cell responses are intact, as can be seen following priming with myelin basic protein in CFA (12). For this reason $C D 40 L^{-/-}$mice provide a useful animal model for clarifying the role of CD40L-CD40 interaction in mucosally induced tolerance.

The effect of CD40L in this response may be partially explained by the involvement of another costimulatory pathway, involving the antigen-presenting cell-borne (APC-borne) B7 proteins (also known as CD80 and CD86) and $\mathrm{T}$ cell-borne counter-receptors CTLA- 4 and CD28. Thus, Samoilova et al. (13) report that blockade of the B7-CTLA-4 interaction can completely abrogate mucosally induced tolerance, whereas blockade of both CD28 and CTLA- 4 interactions with B7 molecules has a similar but less dramatic effect. Hence, a proper costimulatory signal generated by CTLA- 4 is required for mucosally induced tolerance (Figure 1a), whereas CD28 can apparently activate $\mathrm{T}$ cell responses to mucosally derived antigens. In addition, other studies show that stimulation of CD40 with CD40L activates expression of the B7 proteins CD80 and CD86 on the APC surface, thus favoring the interaction of these cells with CD28- or CTLA-4-bearing CD4 T cells (14). 
Consistent with this model, Perez et al. (15) observed that the interaction of the B7 proteins with CTLA-4 provides negative signals that limit $T$ cell activation.

Hence, our finding (9) that the lack of CD40L-CD40 interaction blocks the induction of tolerance could reflect the loss of negative signals provided by CTLA-4-CD80/CD86 interaction. The absence of such an effect following treatment with anti-CD40L mAb, as reported by Hänninen et al. (8), may be explained if the doses used were insufficient to block this negative signal cascade completely (Figure 1b). Alternatively, the discrepant findings may reflect the different developmental histories of the animals studied. In particular, the deletion of the CD4OL gene led to the disorganization and dysfunction of Peyer's patches, organs that are central to the induction of the mucosal immune system (10) and represent an important site for the initiation of oral tolerance (16). The transient antibody blockade used by Hänninen et al. (8), conversely, would not be expected to duplicate this effect on Peyer's patch development.

\section{CD40 L in T cell development}

Perhaps as a result of its proposed effects on the expression of costimulatory molecules, CD40 signaling appears to alter the course of $\mathrm{T}$ cell development. Kumanogoh et al. (17) recently reported that an important regulatory $T$ cell subtype, the $\mathrm{CD}_{25}{ }^{+} \mathrm{CD} 4^{+} \mathrm{CD} 45 \mathrm{RB}^{\text {low }} \mathrm{T}$ cells (shown as $\mathrm{Tr}$ in Figure 1), which can downregulate the pathogenic $\mathrm{CD} 4{ }^{+} \mathrm{CD} 45 \mathrm{RB}$ high $\mathrm{T}$ cell subset, is markedly reduced in $\mathrm{CD}_{40} 0^{-/}$mice. These authors also found that $\mathrm{T}$ cell autoreactivity is significantly increased in these animals. Interestingly, adoptive transfer of T cells isolated from $C D 40^{-/-}$mice can trigger autoimmune diseases in the recipient $n u / n u$ mice in association with enhanced levels of various autoantibodies. Further, APCs isolated from the $C D 40^{-/-}$mice fail to induce regulatory $\mathrm{T}$ cells producing high levels of the suppressive cytokine IL-10. Another group recently showed that a similar population of regulatory $T$ cells can be induced after challenge with oral antigen (18). After alloantigen stimulation, these cells express CD40L at high levels, raising the possibility that CD40L-CD40 interaction a

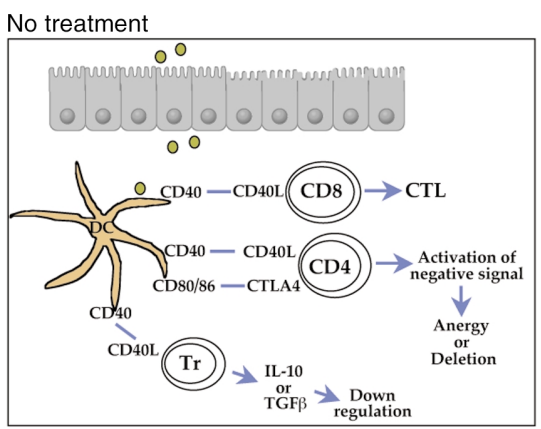

b

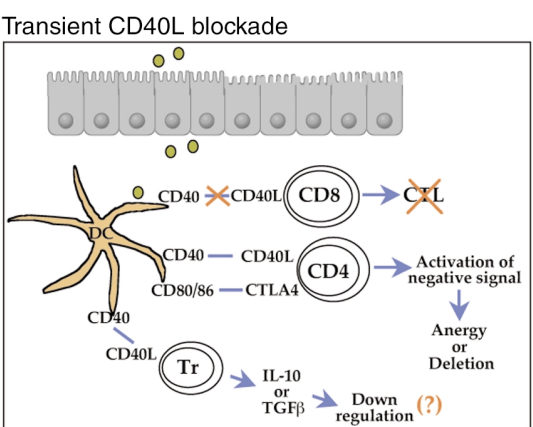

C CD40L deficiency

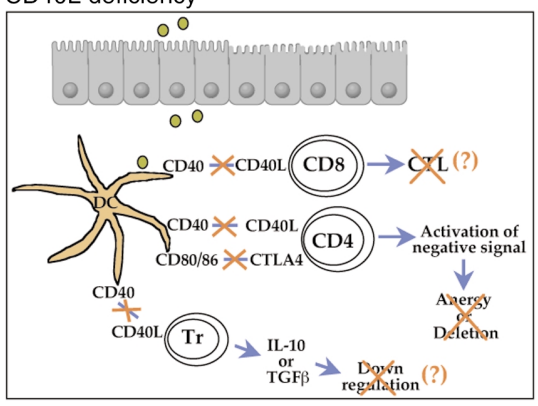

Figure 1

Models for the effects on mucosally induced tolerance of perturbing the CD40L-CD40 interaction. (a) In the untreated wild-type mouse, CD40 on dendritic cells (DCs) and other APCs interacts with CD40L expressed on T cells of several subsets, including CD4 and CD8 cells, as well as a CD25 CD4 population of regulatory $T$ cells $(T r)$. This interaction can lead to both active, antigen-specific CTL responses and the suppression of Th function and inflammation. The latter effects appear to be implicated in mucosally induced tolerance to dietary antigens and endogenous bacteria. (b) As shown by Hänninen et al. in this issue of the $\mathrm{JCl}(8)$, transient treatment with mAb anti-CD40L can prevent induction of CTLs, while apparently leaving some of the other responses intact. Thus, mucosally induced tolerance is not compromised by this treatment. (c) In case of the permanent blockage of CD40L-CD40, as occurs in CD4OL $\mathrm{L}^{-/-}$mice, the additional loss of some immunosuppressive signaling cascade and/or of a population of Tr cells prevents mucosally induced tolerance.

helps regulate their alloantigen-specific immune responses (19). Hence, in the absence of CD40L-CD40 interactions (9), depletion of the population of these regulatory $\mathrm{T}$ cells may reduce the expression of IL-10 and TGF- $\beta$, immunosuppressive cytokines that promote mucosally induced tolerance (Figure 1c). In the case of transient blockade of CD40L-CD40 signaling (8), conversely, the inhibitory signal provided by regulatory $\mathrm{CD} 25^{+} \mathrm{CD}^{+} \mathrm{T}$ cells may remain intact (Figure 1b).

\section{CD40L and CTL responses to mucosal antigens}

Several recent studies show that, in addition to its tolerogenic effect, oral or nasal administration of soluble autoantigens can generate antigenspecific CTLs $(6,20)$. The current report from Hänninen et al. (8) confirms these data and suggests possible mechanisms for the effect on CTL function. CD40L- and CD40-mediated interactions between CD4 T cells and mucosal dendritic cells (DCs) might contribute to this potentially 
harmful response in either of two ways. First, in response to orally administered antigen, soluble factors and/or signaling through CD40L and CD40 could favor the generation of antigen-specific CTLs. Indeed, CD40L/CD40-mediated activation of mucosal DC by antigen-specific CD 4 $T$ cells has been shown to recruit CD8 $\mathrm{T}$ cells into the DC-T cell clusters and to be essential for the subsequent priming of these CD8 T cells (21). Alternatively, since CD8 T cells can express CD40L, mucosal DCs may interact directly with them to promote their activation against specific antigens (22). In this second model, the effect on the CTL response of blocking CD40L, as demonstrated by Hänninen et al. (8), could be explained by a direct effect on CD8 T cells, which might subsequently interact with different DC populations, including those residing in the mucosal compartment.

Recently, several interesting studies have demonstrated a critical and unique role of mucosal DCs in modulating quiescent or aggressive mucosal immune responses that can lead to either mucosally induced tolerance or mucosal immunity (23-25). Manipulation of mucosal DCs could therefore offer novel strategies to induce mucosally induced tolerance without generating active and harmful immune responses, including the generation of autoantigen-specific CTLs. The current work showing that transient blockade of CD40L can dissociate mucosally induced tolerance from the generation of harmful CTLs (Figure $1 \mathrm{~b}$ ) suggests another possible therapeutic strategy that could be applied to autoimmune diseases.
However, because the effects of this procedure clearly differ from the effects of complete CD40L deficiency, fundamental questions about the roles of these molecules remain to be addressed before CD40L-inhibitory drugs or antibodies can be considered for clinical applications.

\section{Acknowledgments}

We thank Kohtaro Fujihashi (University of Alabama at Birmingham) for helpful comments and William R. Brown for editing the Commentary. The results discussed in this Commentary were generated through investigations supported by the Ministry of Education, Science, Sports and Culture and the Ministry of Health and Welfare (Japan) and by the NIH.

1. Strobel, S., and Mowat, A.M. 1998. Immune responses to dietary antigens: oral tolerance. Immunol. Today. 19:173-181.

2. Weiner, H.L. 2000. Oral tolerance, an active immunologic process mediated by multiple mechanisms. J. Clin. Invest. 106:935-937.

3. Faria, A.M., and Weiner, H.L. 1999. Oral tolerance: mechanisms and therapeutic applications. Adv. Immunol. 73:153-264

4. Miller, A., Lider, O., Abramsky, O., and Weiner, H.L. 1994. Orally administered myelin basic protein in neonates primes for immune responses and enhances experimental autoimmune encephalomyelitis in adult animals. Eur. J. Immunol. 24:1026-1032.

5. Weiner, H.L. 1999. Oral tolerance with copolymer 1 for the treatment of multiple sclerosis. Proc Natl. Acad. Sci. USA. 96:3333-3335.

6. Hänninen, A., Braakhuis, A., Heath, W.R., and Harrison, L.C. 2001. Mucosal antigen primes diabetogenic cytotoxic T-lymphocytes regardless of dose or delivery route. Diabetes. 50:771-775.

7. Blanas, E., Carbone, F.R., Allison, J., Miller, J.F., and Heath, W.R. 1996. Induction of autoimmune diabetes by oral administration of autoantigen. Science. 274:1707-1709.

8. Hänninen, A., Martinez, N.R., Davey, G.M., Heath, W.R., and Harrison, L.C. 2002. Transient blockade of CD40 ligand dissociates pathogenic from protective mucosal immunity. J. Clin. Invest. 109:261-267. DOI:10.1172/JCI200213720.

9. Kweon, M.-N., et al. 1999. Mucosally induced sys- temic $\mathrm{T}$ cell unresponsiveness to ovalbumin requires CD40 ligand-CD40 interactions. J. Immunol. 162:1904-1909.

10. Xu, J., et al. 1994. Mice deficient for the CD40 lig and. Immunity. 1:423-431.

11. Renshaw, B.R., et al. 1994. Humoral immune responses in CD40 ligand-deficient mice. J. Exp. Med. 180:1889-1900.

12. Grewal, I.S., et al. 1996. Requirement for CD40 ligand in costimulation induction, $\mathrm{T}$ cell activation, and experimental allergic encephalomyelitis. Science. 273:1864-1867.

13. Samoilova, E.B., et al. 1998. CTLA-4 is required for the induction of high dose oral tolerance. Int. Immunol. 10:491-498.

14. Ranheim, E.A., and Kipps, T.J. 1993. Activated T cells induce expression of $\mathrm{B} 7 / \mathrm{BB} 1$ on normal or leukemic B cells through a CD40-dependent signal. J. Exp. Med. 177:925-935.

15. Perez, V.L., et al. 1997. Induction of peripheral T cell tolerance in vivo requires CTLA-4 engagement. Immunity. 6:411-417.

16. Fujihashi, K., et al. 2001. Peyer's patches are required for oral tolerance to proteins. Proc. Natl. Acad. Sci. USA. 98:3310-3315.

17. Kumanogoh, A., et al. 2001. Increased T cell autoreactivity in the absence of CD40-CD40 lig and interactions: a role of CD40 in regulatory T cell development. J. Immunol. 166:353-360.

18. Zhang, X., Izikson, L., Liu, L., and Weiner, H.L. 2001. Activation of $\mathrm{CD} 25^{+} \mathrm{CD}^{+}$regulatory T cells by oral antigen administration. J. Immunol. 167:4245-4253

19. Taylor, P.A., Noelle, R.J., and Blazar, B.R. 2001 $\mathrm{CD} 4{ }^{+} \mathrm{CD} 25^{+}$immune regulatory cells are required for induction of tolerance to alloantigen via costimulatory blockade. J. Exp. Med. 193:1311-1318.

20. Kim, S.K., et al. 1998. Generation of mucosa cytotoxic T cells against soluble protein by tissuespecific environmental and costimulatory signals. Proc. Natl. Acad. Sci. USA. 95:10814-10819.

21. Ridge, J.P., Di Rosa, F., and Matzinger, P. 1998. A conditioned dendritic cell can be a temporal bridge between a CD4 ${ }^{+}$T-helper and a T-killer cell. Nature. 393:474-478.

22. Lefrancois, L., Olson, S., and Masopust, D. 1999. A critical role for CD40-CD40 ligand interactions in amplification of the mucosal CD8 $\mathrm{T}$ cell response. J. Exp. Med. 190:1275-1284.

23. Banchereau, J., and Steinman, R.M. 1998. Den dritic cells and the control of immunity. Nature. 392:245-252.

24. Iwasaki, A., and Kelsall, B.L. 1999. Mucosa immunity and inflammation. I. Mucosal dendritic cells: their specialized role in initiating $\mathrm{T}$ cell responses. Am. J. Physiol. 276:G1074-G1078.

25. Kelsall, B.L., and Strober, W. 1996. Distinct populations of dendritic cells are present in the subepithelial dome and $\mathrm{T}$ cell regions of the murine Peyer's patch. J. Exp. Med. 183:237-247. 